\title{
Refinement and verification of concurrent systems specified in Object-Z and CSP
}

\author{
Graeme Smith* and John Derrick $\dagger$ \\ * Technische Universität Berlin, FB Informatik, FG Softwaretechnik, \\ Sekr. FR 5-6, Franklinstr. 28/29, D-10587 Berlin, Germany. \\ $\dagger$ Computing Laboratory, University of Kent, Canterbury, CT2 7NF, UK. \\ (Email: graeme@cs.tu-berlin.de and J.Derrick@ukc.ac.uk.)
}

\begin{abstract}
The formal development of large or complex systems can often be facilitated by the use of more then one formal specification language. Such a combination of languages is particularly suited to the specification of concurrent or distributed systems, where both the modelling of processes and state is necessary. This paper presents an approach to refinement and verification of specifications written using a combination of Object-Z and CSP.

A common semantic basis for the two languages enables a unified method of refinement to be used, based upon CSP refinement. To enable state-based techniques to be used for the Object-Z components of a specification we develop state-based refinement relations which are sound and complete with respect to CSP refinement.

In addition, a verification method for static and dynamic properties is presented. The method allows us to verify properties of the CSP system specification in terms of its component Object-Z classes by using the laws of the CSP operators together with the logic for Object-Z.
\end{abstract}

Keywords: Object-Z; CSP; Refinement; Verification; Concurrency.

\section{Introduction}

The formal development of particularly large, or complex, systems can often be facilitated by the use of more then one formal specification language. While most specification languages can be used to specify entire systems, few, if any, are particularly suited to modelling all aspects of such systems. This realisation has lead to the development of new specification languages which combine features of one or more existing languages $[1,8]$ and, more recently, approaches for formally integrating existing languages $[4,24,11,22,9]$.

Such a combination of languages is particularly suited to the specification of concurrent or distributed systems, where both the modelling of processes and state is necessary. Process algebras such as CCS[16] and CSP[12] are suitable vehicles for modelling the interactions between processes or their temporal ordering. State-based languages such as Z[23] or VDM[14], however, offer better facilities for the specification of the complex data structures which may be needed to describe the processes themselves. Indeed, the Open Distributed Processing reference model[13] recognises that different languages are likely to be used in the different viewpoint specifications of a large distributed system. 
A method of formally specifying concurrent systems using Object-Z[7], an object-oriented extension of $\mathrm{Z}$, together with CSP is described in [22]. The rationale is that Object- $\mathrm{Z}$ provides a convenient method of modelling the complex data structures needed to define component processes, and CSP enables the concise specification of process interaction. The advantage of Object- $Z$ over more traditional state-based languages such as $\mathrm{Z}$ is that its class structure provides a construct easily identifiable with CSP processes. The basis of the integration is a semantics of Object- Z classes identical to that of CSP processes. This enables classes specified in Object-Z to be used directly within the CSP part of the specification.

However, in addition to specification, a notation needs to be able to support incremental development of specifications through a well-defined method of refinement. It is also desirable to be able to verify both static and dynamic, i.e. behavioural, properties of these specifications. The work described here presents a method of refining specifications written in the integrated Object-Z / CSP notation, and a method for verifying such properties of those specifications.

The common semantic basis for the two languages enables a unified method of refinement to be developed for the integrated notation: because we give Object-Z classes a CSP semantics, we can use CSP refinement as the refinement relation for the integrated notation. However, as a means for verifying a refinement it is more convenient to be able to use a state-based refinement relation for the Object- $\mathrm{Z}$ components, rather than having to calculate their semantics. In order to do so, we adapt the work of Josephs[15], who has developed refinement relations for state-based systems which are sound and complete with respect to CSP refinement.

In order to be able to verify static and dynamic properties, we present a method of verification for the integrated notation. The method allows us to verify properties of the CSP system specification in terms of its component Object-Z classes by using the laws of the CSP operators presented in [12] together with the logic for Object-Z in [19]. CSP and Object-Z properties are related via auxiliary variables introduced into the Object-Z classes using inheritance.

The paper is structured as follows. Section 2 presents the integration of Object-Z and CSP based on the common semantics. Section 3 then discusses refinement in the integrated notation, and defines the state-based refinement relations that we will use for the Object- $Z$ components of a specification. Section 4 explains how properties of specifications can be verified, and we conclude in Section 5. Throughout the paper we illustrate these techniques with the specification and refinement of a cinema booking system.

\section{Integrating Object-Z and CSP}

This section presents the integration of Object-Z and CSP. The basis of this integration is a semantics of Object-Z classes identical to that of CSP processes. This allows classes specified in Object-Z to be used directly within the CSP part of the specification. The approach to specification comprises three phases.

- The first phase involves specifying the components of the system using Object-Z. Since all interaction of system components is specified in the CSP part of the specification, a restricted subset of Object-Z is used which does not include instantiation of objects of a class (see [7] for details). This restriction greatly simplifies reasoning about the Object-Z part of the specification.

- The components specified in the first phase will generally not be in a form that allows them to be composed using CSP operators. The second phase involves modifying the class interfaces so that they will synchronise and communicate as desired. This may be achieved using Object-Z inheritance. 
This optional phase is not required for the simple examples presented in this paper. An example illustrating its use can be found in [22].

- The final phase involves the specification of the system using CSP operators. As detailed in this section, a well-definedness condition is placed on the hiding operator restricting its use.

To illustrate the approach we present a case study of a cinema booking system. This case study is based on the specification of the Apollo box office in [25] but extended to support multiple customers.

\subsection{Specifying the components of a system}

The Marlowe box office allows customers to book tickets in advance by telephone. When a customer calls, if there is an available ticket then one is allocated and put to one side for the caller. When the customer arrives, they are presented with this ticket.

The components of the booking system are the customers and the Marlowe box office. In our approach, these will be specified by Object-Z classes. A class in Object-Z is represented syntactically by a named box possibly with generic parameters. In this box there may be local type and constant definitions, at most one state schema and associated initial state schema, and zero or more operation schemas. As an example, consider the specification of a customer of the booking system.

Let Name denote the set of all customer names and Ticket the set of all tickets.

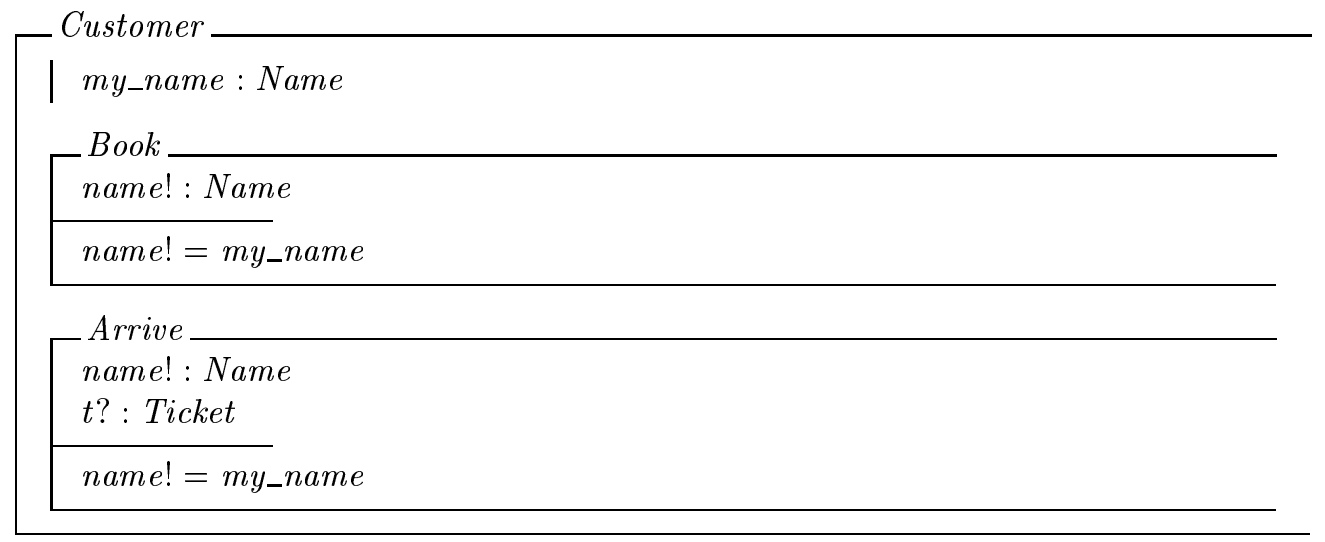

This class has a single constant my_name denoting the name of the customer and two operations: Book and Arrive. The operations Book and Arrive correspond to the customer booking a ticket and arriving to collect a ticket respectively. They have input parameters (denoted by names ending in ?) and output parameters (denoted by names ending in !) for communication with the box office.

A more substantial example of a class is provided by the specification of the Marlowe box office. 


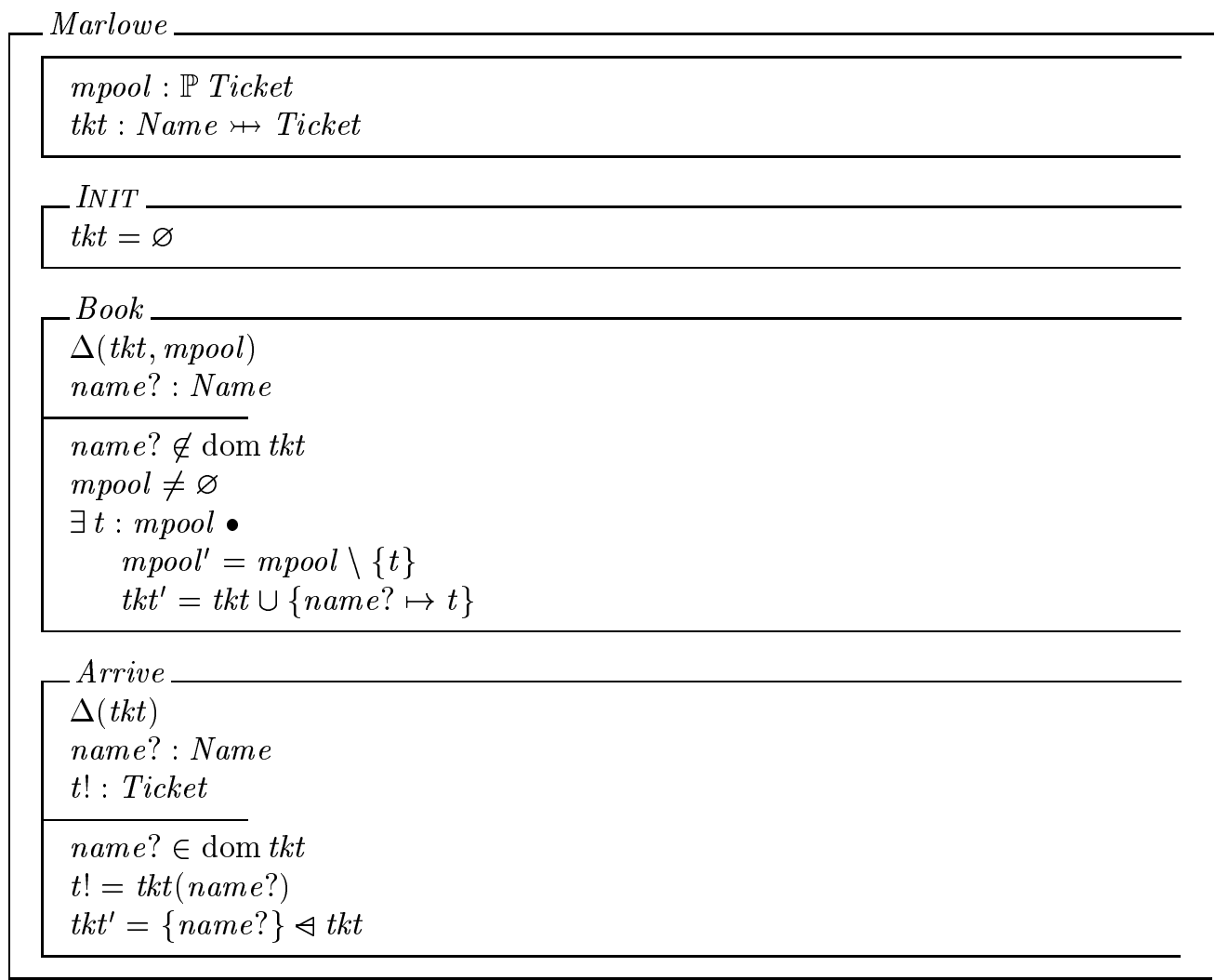

This class has a state schema with two state variables: mpool, denoting the pool of tickets, and $t k t$, a partial injective function from Name to Ticket recording which tickets have been allocated to which customers. Initially, no tickets have been allocated.

Each operation schema has a $\Delta$-list of the state variables which it may change. State variables not listed remain unchanged. The operation Book is feasible whenever there are still tickets available $($ mpool $\neq \varnothing)$ and allocates a ticket to a customer who has not already made a booking (name? $\notin$ dom $t k t$ ). The operation Arrive issues the ticket but does not change the pool of tickets (mpool $=$ mpool $^{\prime}$ is a consequence of mpool not appearing in the $\Delta$-list of the operation Arrive).

\section{$2.2 \quad$ Specifying the system}

To specify the booking system we use CSP operators to capture the interaction between the customers and box office. This is made possible by giving a semantics to Object-Z classes which is identical to that of CSP processes.

\subsubsection{Semantics of CSP processes}

There are several semantic models for CSP processes. The most widely accepted of these is the failures-divergences semantics of $[3,12]$. In this semantics, a process is modelled by the triple $(A, F, D)$ where $A$ is its alphabet (i.e. the set of events that it can possibly engage in $)^{1}, F$ is its failures and $D$ its divergences. The failures of a process are pairs $(s, X)$ where $s$ is a trace of the process, i.e. a finite sequence of events that the process may undergo, and $X$ is a set of events the

\footnotetext{
${ }^{1}$ The alphabet is made implicit in [3] by assuming all processes have the same alphabet.
} 
process may refuse to perform after undergoing $s$. That is, if the process after undergoing $s$ is in an environment which only allows it to undergo events in $X$, it may deadlock. The divergences of a process are the sequences of events after which the process may undergo an infinite sequence of internal events, i.e. livelock. Divergences also result from unguarded recursion.

We adopt, however, a variant of the simpler failures semantics of [2]. This semantics doesn't include a component corresponding to the divergences of a process. The reason for adopting this simpler semantics is because Object- $\mathrm{Z}$ is capable of modelling unbounded nondeterminism, i.e. where a choice is made from an infinite set, which cannot be modelled in standard CSP. As shown in [17] and [22], this can lead to problems when calculating divergences.

Given a class with alphabet $A$ and failures $F \subseteq A^{*} \times \mathbb{P} A$, the properties of the semantics we adopt are as follows.

$$
\begin{aligned}
& (\langle\rangle, \varnothing) \in F \\
& (s \frown t, \varnothing) \in F \Rightarrow(s, \varnothing) \in F \\
& (s, X) \in F \wedge Y \subseteq X \Rightarrow(s, Y) \in F \\
& (s, X) \in F \wedge(\forall x \in Y \bullet(s \frown\langle x\rangle, \varnothing) \notin F) \Rightarrow(s, X \cup Y) \in F
\end{aligned}
$$

That is, we have dropped the restriction in [2] that the set of refused events is finite as is also done in $[3]^{2}$ and $[15]$.

For the failures semantics to be adequate, however, we must ensure that our specifications are divergence free. This is true of processes corresponding to Object- $\mathrm{Z}$ classes since Object- $\mathrm{Z}$ has no notion of internal operations nor recursive definitions of operations ${ }^{3}$. It can be ensured for other processes in our approach by placing a well-definedness condition on the hiding operator of CSP as is done in [15]. That is, given a process $P$ with failures $F, P \backslash C$ is well-defined only if

$$
\forall s \in \operatorname{dom} F \bullet \neg\left(\forall n \in \mathbb{N} \bullet \exists t \in C^{*} \bullet \# t>n \wedge s \frown t \in \operatorname{dom} F\right)
$$

This prevents infinite sequences of events being hidden.

An alternative solution to the problem of unbounded nondeterminism would be to add to the failures-divergences semantics a component corresponding to the infinite traces of a process as is done in [18]. In this case, no restriction would be required on hiding. Whether the benefits of adopting this more complicated semantics are worthwhile, however, needs to be investigated.

\subsubsection{Semantics of Object-Z classes}

A semantics of Object-Z classes is presented in [21] where, following the work of [6], a class is modelled by its set of histories, i.e. the sequences of states it can pass through together with the corresponding sequences of operations it can undergo.

Given the set of all possible identifiers $I d$ and the set of all possible values Value, the states of a class can be represented by a set

$$
S \subseteq(I d \rightarrow \text { Value })
$$

and the operations by a set

\footnotetext{
${ }^{2}$ The additional property stating that a set is refusable if all its finite subsets are refusable in [3] was shown to be unnecessary in [17].

${ }^{3}$ Although recursive definitions of operations have been suggested for Object-Z (e.g. [5]), we have adopted a more conservative view of Object-Z in this paper.
} 


$$
O \subseteq I d \times(I d \pitchfork \text { Value })
$$

The operations are instances of the class' operation schemas. They comprise the name of the operation schema together with an assignment of values to its parameters. For example, $($ Book, $\{($ name?,$n)\})$ where $n \in$ Name is a possible operation of the class Marlowe.

A history is a non-empty sequence of states together with a sequence of operations. Either both sequences are infinite ${ }^{4}$ or the state sequence is one longer than the operation sequence. The histories of a class with states $S$ and operations $O$ can be represented by a set

$$
H \subseteq S^{\omega} \times O^{\omega}
$$

such that the following properties hold.

$$
\begin{aligned}
& (s, o) \in H \Rightarrow s \neq\langle\rangle \\
& (s, o) \in H \wedge s \notin S^{*} \Rightarrow o \notin O^{*} \\
& (s, o) \in H \wedge s \in S^{*} \Rightarrow \# s=\# o+1 \\
& \left(s_{1} \frown s_{2}, o_{1} \frown o_{2}\right) \in H \wedge \# s_{1}=\# o_{1}+1 \Rightarrow\left(s_{1}, o_{1}\right) \in H
\end{aligned}
$$

The first three properties capture the requirements on an individual history detailed above. The final property is a condition on the set of histories representing a class. This set must be prefixclosed. This is necessary since any prefix of a class' history also represents a possible evolution of the class.

\subsubsection{Modelling classes as processes}

In order to relate classes and processes, we need to relate operations and events. This needs to be done in such a way that appropriate input and output parameters of synchronising operations can be identified. We therefore define a meta-function $\beta$ which returns the basename of a parameter name, i.e. $\beta(x ?)=\beta(x !)=x$, and allow it be applied to the assignment of values to an operation's parameters as follows.

$$
\begin{gathered}
\beta\left(\left\{\left(x_{1}, v_{1}\right), \ldots,\left(x_{n}, v_{n}\right)\right\}\right)=\left\{\left(\beta\left(x_{1}\right), v_{1}\right), \ldots,\left(\beta\left(x_{n}\right), v_{n}\right)\right\} \\
\text { where }\left\{x_{1}, \ldots, x_{n}\right\} \subseteq I d \text { and }\left\{v_{1}, \ldots, v_{n}\right\} \subseteq \text { Value }
\end{gathered}
$$

The function relating operations and events is then defined as follows.

$$
\text { event }((n, p))=n . \beta(p) \quad \text { where } n \in I d \text { and } p \in(I d m \text { Value })
$$

For example, the event corresponding to a customer with name $n$ making a booking is Book. $\{($ name, $n)\}$. This event is identical to that corresponding to the box office accepting a booking from a customer with name $n$. Hence, these two operations will be able to synchronise when their classes are combined using the CSP parallel composition operator $\|$. Similarly, the events corresponding to a customer with name $n$ arriving and collecting a ticket $s$ and the box office allocating ticket $s$ to that customer will be the event Arrive. $\{($ name,$n),(t, s)\}$.

We let a class $C$ be modelled by a parameterised process $C_{i}$. The parameter $i$ is an assignment of values to a subset of the state of $C$ satisfying a possible initial state of $C$. That is, $i \in\{j \mid$

\footnotetext{
${ }^{4}$ Infinite histories enable liveness properties of classes to be modelled. Such properties have been ignored in the description of Object-Z in this paper.
} 
$\exists(s, o) \in H \bullet j \subseteq s(1)\}^{5}$. This allows us to refer to the class' constants when it is used as a process. For example, we can define a process Customer $_{n}$ corresponding to the customer with name $n$ as follows.

$$
\text { Customer }_{n}=\text { Customer }_{\left\{\left(m y \_n a m e, n\right)\right\}}
$$

For notational convenience, we introduce the convention that $C=C_{\varnothing}$ allowing us to write, for example, Marlowe rather than Marlowe $\varnothing$ for the process corresponding to the class Marlowe without any restriction on the initial state.

Given a class $C$ with states $S$, operations $O$ and histories $H$, the alphabet of process $C_{i}$ comprises the events corresponding to the operations in $O$.

$$
\operatorname{alphabet}\left(C_{i}\right)=\{\operatorname{event}(o p) \mid o p \in O\}
$$

To define the failures of a class we use the following function which maps a sequence of operations to a sequence of events.

$$
\begin{aligned}
& \text { events }(\langle\rangle)=\langle\rangle \\
& \text { events }(\langle o p\rangle \frown o)=\langle\operatorname{event}(o p)\rangle \frown \operatorname{events}(o)
\end{aligned}
$$

The failures of $C_{i}$ are derived from the histories in $H$ as follows: $(t, X)$ is a failure of $C_{i}$ if

- there exists a finite history of $C$ whose initial state is satisfied by $i$,

- the sequence of operations of the history corresponds to the sequence of events in $t$ and

- for each event in $X$, there does not exist a history which extends the original history by an operation corresponding to that event.

$$
\begin{aligned}
\text { failures }\left(C_{i}\right)=\{(t, X) \mid \exists(s, o) \in H \bullet & \\
& s \in S^{*} \wedge \\
& i \subseteq s(1) \wedge \\
& t=\operatorname{events}(o) \wedge \\
& \forall e \in X \bullet \nexists \operatorname{st} \in S, o p \in O \bullet \\
& \quad e=\operatorname{event}(o p) \wedge(s \frown\langle s t\rangle, o \frown\langle o p\rangle) \in H\}
\end{aligned}
$$

As shown in [22], the failures of $C_{i}$ defined in this way satisfy the properties $F 1$ to $F 4$ of the failures semantics.

\subsubsection{The booking system specification}

The processes Customer $_{n}$ and Marlowe can now be composed to specify the booking system.

$$
\text { BookingSystem }=\left(\|\|_{n: \text { Name }} \text { Customer }_{n}\right) \| \text { Marlowe }
$$

That is, the booking system consists of the box office running concurrently with a collection of customers - one for each name in Name. Since this part of the specification is a CSP specification,

\footnotetext{
${ }^{5}$ An Object-Z class with unsatisfiable initial constraints is not given a semantics in this approach. Such degenerate classes are, however, unimplementable and of no practical interest to the specifier.
} 
we can state properties we wish to prove about it in the same way as they are stated in CSP (see [12]). That is, in the form $P$ sat $S$ where $P$ is a process and $S$ is a predicate in terms of $t r$, the traces, and ref, the refusal sets, of the failures of process $P$. For example, the property that the number of bookings made is greater than or equal to the number of tickets allocated to arriving customers can be stated as follows ${ }^{6}$.

BookingSystem sat \#tr $\downarrow$ Book $\geqslant \# t r \downarrow$ Arrive

An approach to proving such properties in terms of the component Object-Z classes is presented in Section 4.

\section{$3 \quad$ Refining Object-Z and CSP specifications}

This section presents a method of refinement for systems specified using the integrated Object-Z / CSP notation. The use of a CSP semantics for Object-Z classes enables us to use CSP refinement as the refinement relation for the integrated notation. To verify such a refinement there are two different approaches that can be employed:

- The first is based on the approach used in CSP. The refinement is verified directly by calculating and comparing the failures of the specifications or, in the case where the specifications have identical structure, the failures of the components of the specifications.

- The second involves using state-based methods to verify the refinement of the component Object-Z classes of a specification. This is achieved by adapting the work of Josephs[15], which provides refinement relations for state-based systems that are sound and complete with respect to CSP refinement. This approach is only possible when the specifications have identical structure.

In this section we illustrate both approaches by refining the cinema booking system of Section 2 .

\subsection{Failures Approach}

Refinement in CSP is defined in terms of failures and divergences[3]. A process $Q$ is a refinement of a process $P$ if

$$
\text { failures } Q \subseteq \text { failures } P \text { and divergences } Q \subseteq \text { divergences } P
$$

or when using the simpler failures semantics if

$$
\text { failures } Q \subseteq \text { failures } P \text {. }
$$

We write $P \sqsubseteq Q$ to denote the latter. Because we have modelled Object-Z classes semantically as processes, CSP refinement can be used as the basis for refining specifications written in the integrated Object-Z / CSP notation. As an example, consider an alternative booking system to the BookingSystem specification given in Section 2.

Like the Marlowe box office, the Kurbel box office allows customers to book tickets in advance by telephone. However, the procedure is different from that used at the Marlowe. When a customer calls, if there is an available ticket then the customer's name is simply recorded. When a customer whose name has been recorded arrives at the box office, a ticket is allocated.

\footnotetext{
${ }^{6} s \downarrow c$ denotes the sequence of values $v$ of events of the form $c . v$ in $s$, e.g. $\langle c .1, a .4, c .3, d .1\rangle \downarrow c=\langle 1,3\rangle$.
} 
The contrast between the Marlowe and the Kurbel box offices is the point of allocation of tickets (at booking time vs at collection time). However, at this level of abstraction the customer cannot tell that the Kurbel is behaving differently to the Marlowe. We will prove this property by showing that the Kurbel booking system is a CSP refinement of the Marlowe booking system.

The components of the Kurbel booking system are the customers and the Kurbel box office. The specification of a customer is identical to that given in the Marlowe booking system. The Kurbel box office is represented by the following Object-Z class.

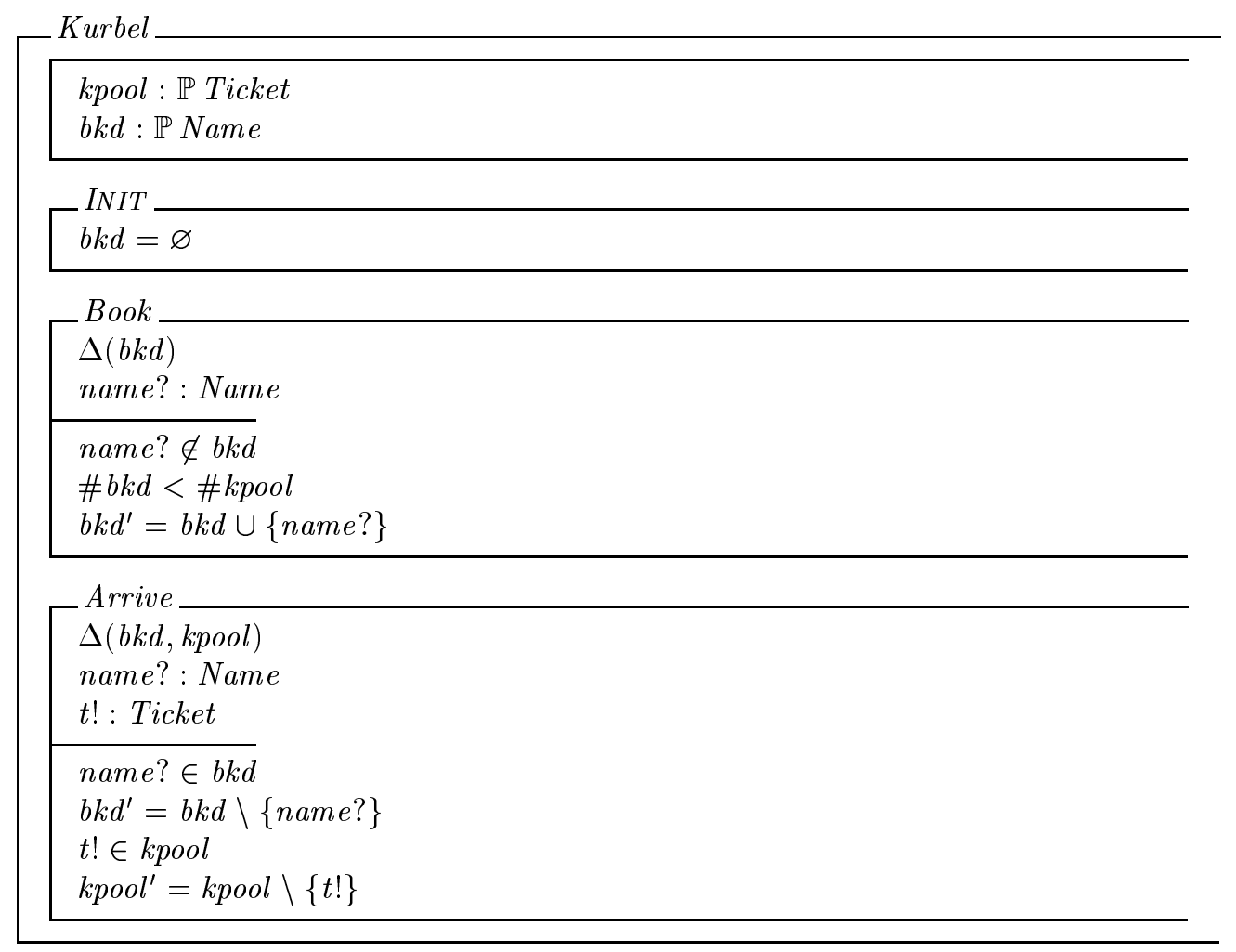

The state variable kpool denotes the pool of tickets and $b k d$ denotes the set of names of customers who have booked a ticket. Initially, $b k d$ is empty. The operation Book records a booking provided that there are currently less bookings than tickets and, hence, still tickets available. The operation Arrive allocates a ticket to a customer who has a booking.

The complete system again consists of the box office running concurrently with a collection of customers.

$$
\text { BookingSystem }_{K}=\left(\mid \|_{n: \text { Name }} \text { Customer }_{n}\right) \| \text { Kurbel }
$$

To show that BookingSystem ${ }_{K}$ is a refinement of BookingSystem, we will compare their failures. Since the structure of the booking system specifications are identical and the components Customer $_{n}$ are identical, we need only show that failures(Kurbel) $\subseteq$ failures(Marlowe).

Consider first the class Kurbel. The failures of Kurbel can be given in terms of the failures of the processes $\left.K_{u r b e}(k p o o l, p)\right\}$ for each possible set of tickets $p$.

$$
\text { failures }(\text { Kurbel })=\bigcup_{p \in \mathbb{P} \text { Ticket }} \text { failures }\left(\text { Kurbel }_{\{(\text {kpool }, p)\}}\right)
$$

The traces of $K_{\text {urbe }} l_{\{(k p o o l, p)\}}$ comprise the empty trace and any trace formed by extending a trace of $\left.K_{u r b e l}(k p o o l, p)\right\}$ by 
- a Book event whenever the customer doing the booking has arrived and collected any tickets he or she has previously booked and

- an Arrive event whenever

- the ticket being collected was initially in kpool,

- the ticket being collected has not been previously collected by any customer and

- the customer arriving has booked once more than he or she has arrived to collect a ticket.

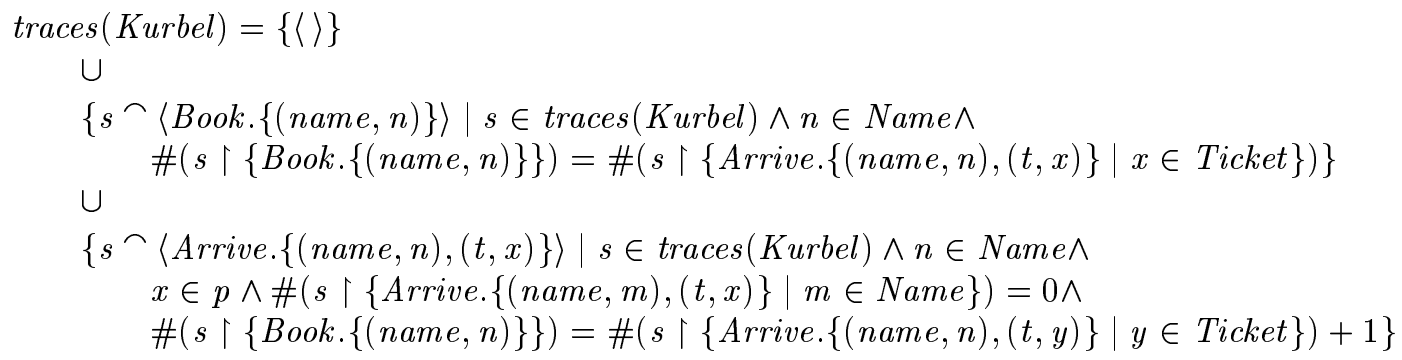

Kurbel $_{\{(k p o o l, p)\}}$ can refuse a Book event whenever the customer making the booking has booked more times than he or she has arrived, or there are no tickets remaining in kpool. It can refuse an Arrive event whenever the customer arriving has already arrived as many times as he or she has booked, the ticket of the Arrive event has already been allocated to a customer or the ticket of the Arrive event was not in kpool initially.

Hence, the failures of $\operatorname{Kurbel}_{\{(k p o o l, p)\}}$ are

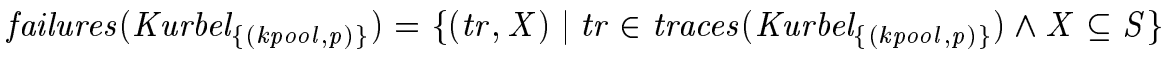

$$
\begin{aligned}
& \text { where } \\
& S=\{\text { Book. }\{(\text { name }, n)\}, \text { Arrive. }\{(\text { name }, m),(t, x)\} \mid x \in \text { Ticket } \wedge n, m \in \text { Name } \wedge \\
& (\#(\operatorname{tr}\lceil\{\text { Book. }\{(\text { name }, n)\}\}>\#(\operatorname{tr}\lceil\{\text { Arrive. }\{(\text { name }, n),(t, y) \mid y \in \text { Ticket }\}) \\
& \vee \#(\operatorname{tr}\lceil\{\text { Arrive. }\{(\text { name }, l),(t, y) \mid l \in \text { Name } \wedge y \in \text { Ticket }\}=\# p) \\
& (\#(\operatorname{tr}\lceil\{\text { Book. }\{(\text { name }, m)\}\})=\#(\operatorname{tr}\lceil\{\text { Arrive. }\{(\text { name }, m),(t, x)\}\}) \\
& \vee \#(\operatorname{tr}\lceil\{\text { Arrive. }\{(\text { name }, l),(t, x)\} \mid l \in \text { Name }\}) \neq 0 \\
& \vee x \notin p\} \text {. }
\end{aligned}
$$

The failures of Marlowe can similarly be given in terms of the failures of the processes Marlowe $\{($ mpool,$p)\}$ for each possible set of tickets $p$.

$$
\text { failures }(\text { Marlowe })=\cup_{p \in \mathbb{P} \text { Ticket }} \text { failures }\left(\operatorname{Marlowe}_{\{(\text {mpool }, p)\}}\right)
$$

It easy to see that the traces of $\operatorname{Marlowe}_{\{(m p o o l, p)\}}$ are identical to those of $\operatorname{Kurbel}_{\{(k p o o l, p)\}}$. Furthermore, Marlowe $\{(m p o o l, p)\}_{\text {con }}$ can refuse any events that $\operatorname{Kurbel}_{\{(k p o o l, p)\}}$ can refuse after the same trace. It can, in fact, refuse more events after a given trace because it can refuse an Arrive event whenever the ticket of the Arrive event is not that previously allocated to the customer. Hence, failures $\left(\right.$ Kurbel $_{\left.\{(\text {mpool }, k)\}_{1}\right) \subset \text { failures }}\left(\right.$ Marlowe $\left._{\{(m p o o l, k)\}}\right)$ and, therefore, failures $($ Kurbel $) \subset$ failures $($ Marlowe $)$ as desired. 


\subsection{State-based Approach}

Calculating and comparing the failures of classes as illustrated above is feasible, but can be complex for non-trivial specifications. The purpose of this section is to show how we can use state-based refinement techniques for the Object- $Z$ component of a specification. This will enable refinements to be verified at the specification level, rather than working explicitly in terms of failures, traces and refusals at the semantic level.

Work on state-based refinement for concurrent systems goes back to He[10] and Josephs[15], who have developed refinement relations for state-based transition systems which are complete and sound with respect to CSP refinement. Woodcock and Morgan[27] have produced similar results in the context of action systems and weakest precondition formulae. In this section we adapt the work of Josephs to the Object-Z setting. This work is directly applicable to this context because it uses the failures semantics (as opposed to the failures-divergences model) and places the same restrictions on hiding that we have adopted. We produce two refinement relations, called upward and downward simulation, which together are sound and complete with respect to CSP refinement. Using these rules we can refine the Object-Z components of an integrated Object-Z / CSP specification such that the entire specification is also refined.

Josephs considers a state-based system $P$ to be defined by a tuple $(A, S, \longrightarrow, R)$ where $A$ is its alphabet, $S$ its states, $\longrightarrow$ its transition relation and $R$ its initial states $(R \subseteq S, R \neq \varnothing)$. As usual we will denote a transition under event $e$ from state $\sigma_{1}$ to $\sigma_{2}$ by $\sigma_{1} \stackrel{e}{\longrightarrow} \sigma_{2}$. In addition, the set of next possible events that a system $P$ can undergo when in state $\sigma$ is denoted $\operatorname{next}_{P}(\sigma)$, i.e.

$$
\operatorname{next}_{P}(\sigma)=\left\{e \in A \mid \exists \sigma^{\prime} \in S \bullet \sigma \stackrel{e}{\rightarrow} \sigma^{\prime}\right\}
$$

Refinement in state-based systems is based on the concept of simulations. For example, simulation forms the basis of the refinement rules in $\mathrm{Z}$ as they are usually presented[25]. Josephs uses two versions called downward and upward simulation (sometimes called forward and backward simulations respectively) defined as follows.

Definition 1 Downward simulation $P_{2}$ is a downward simulation of $P_{1}$ if there is a relation $D \subseteq S_{1} \times S_{2}$ such that

1. $\forall \sigma_{1} \in S_{1}, \sigma_{2} \in S_{2} \bullet \sigma_{1} D \sigma_{2} \Longrightarrow \operatorname{next}_{P_{1}}\left(\sigma_{1}\right)=\operatorname{next}_{P_{2}}\left(\sigma_{2}\right)$

2. $\forall \sigma_{1} \in S_{1}, \sigma_{2}, \sigma_{2}^{\prime} \in S_{2}, e \in A \bullet \sigma_{1} D \sigma_{2} \wedge \sigma_{2} \stackrel{e}{\longrightarrow}_{2} \sigma_{2}^{\prime} \Longrightarrow \exists \sigma_{1}^{\prime} \in S_{1} \bullet \sigma_{1} \stackrel{e}{\longrightarrow}{ }_{1} \sigma_{1}^{\prime} \wedge \sigma_{1}^{\prime} D \sigma_{2}^{\prime}$

3. $\forall \sigma_{2} \in R_{2} \bullet \exists \sigma_{1} \in R_{1} \bullet \sigma_{1} D \sigma_{2}$

Definition 2 Upward simulation $P_{2}$ is an upward simulation of $P_{1}$ if there is a relation $U \subseteq S_{1} \times S_{2}$ such that

1. $\forall \sigma_{2} \in S_{2} \bullet \exists \sigma_{1} \in S_{1} \bullet \sigma_{1} U \sigma_{2} \wedge \operatorname{next}_{P_{1}}\left(\sigma_{1}\right) \subseteq \operatorname{next}_{P_{2}}\left(\sigma_{2}\right)$

2. $\forall \sigma_{1}^{\prime} \in S_{1}, \sigma_{2}, \sigma_{2}^{\prime} \in S_{2}, e \in A \bullet \sigma_{1}^{\prime} U \sigma_{2}^{\prime} \wedge \sigma_{2} \stackrel{e}{\longrightarrow}_{2} \sigma_{2}^{\prime} \Longrightarrow \exists \sigma_{1} \in S_{1} \bullet \sigma_{1} \stackrel{e}{\longrightarrow}{ }_{1} \sigma_{1}^{\prime} \wedge \sigma_{1} U \sigma_{2}$

3. $\forall \sigma_{1} \in S_{1}, \sigma_{2} \in R_{2} \bullet \sigma_{1} U \sigma_{2} \Longrightarrow \sigma_{1} \in R_{1}$.

Josephs then proves that these two relations are sound and complete with respect to CSP refinement. 
To use these results, we first adapt the definitions to the Object- $Z$ setting. The translation is straightforward, and the relations $D$ and $U$ between the state spaces are re-cast as retrieve relations (denoted Abs) between the abstract state (Astate) and the concrete state (Cstate).

To translate the rules involving $\operatorname{next}_{P}(\sigma)$ we introduce a new precondition operator Pre. This is necessary because when we model Object- $\mathrm{Z}$ classes as processes we relate operations to events by removing the decorations? and !. Therefore the simulation rules presented above will treat outputs in the same way as inputs. This is in contrast to standard $\mathrm{Z}$ refinement where the constraints on inputs can be weakened and those on outputs strengthened[25]. Doing this in our notation would mean that we could reduce the events that occur under a refinement, and hence restrict possible synchronisation with other processes. Compositionality would then be lost.

So in order to reflect the above simulation rules accurately and maintain compositionality in the Object-Z setting, we define Pre to hide the post-state of an operation, but not its outputs, i.e. Pre $O p \hat{=} \exists$ State $^{\prime} \bullet O p$. The event corresponding to an Object-Z operation $O p$ is in $\operatorname{next}_{P}(\sigma)$ iff Pre $O p$ is true in the state representing $\sigma$. This is because the interpretation of operations in Object-Z differs from that in $\mathrm{Z}$ in that an operation cannot occur when its precondition is not enabled $^{7}$. We can now give the definition of downward and upward simulation in Object-Z.

Definition 3 Downward simulation

An Object- $Z$ class $C$ is a downward simulation of the class $A$ if there is a retrieve relation Abs such that every abstract operation $A O p$ is recast into a concrete operation COp and the following hold.

DS.1 $\forall$ Astate; Cstate $\bullet$ Abs $\Longrightarrow($ Pre $A O p \Longleftrightarrow$ Pre $C O p)$

DS.2 $\forall$ Astate; Cstate; Cstate $^{\prime} \bullet A b s \wedge C O p \Longrightarrow \exists$ Astate $^{\prime} \bullet A b s^{\prime} \wedge A O p$

DS.3 $\forall$ Cinit $\bullet \exists$ Ainit $\bullet$ Abs

Definition 4 Upward simulation

An Object- $Z$ class $C$ is an upward simulation of the class $A$ if there is a retrieve relation Abs such that every abstract operation $A O p$ is recast into a concrete operation COp and the following hold.

US.1 $\forall$ Cstate $\bullet \exists$ Astate $\bullet$ Abs $\wedge$ Pre $A O p \Longrightarrow$ Pre $C O p$

US.2 $\forall$ Astate $^{\prime} ;$ Cstate $;$ Cstate $^{\prime} \bullet C O p \wedge A b s^{\prime} \Longrightarrow \exists$ Astate $\bullet$ Abs $\wedge A O p$

US.3 $\forall$ Astate; Cinit $\bullet$ Abs $\Longrightarrow$ Ainit

Using these rules we can show that the Kurbel class is an upward simulation, and hence a refinement, of the Marlowe class without having to calculate the failures. To do so we first record the relationship between the two classes as a retrieve relation given by

\begin{tabular}{l} 
Ret \\
Kurbel.STATE \\
Marlowe.STATE \\
\hline$b k d=\operatorname{dom} t k t$ \\
$k p o o l=m p o o l \cup \operatorname{ran} t k t$ \\
$m p o o l \cap \operatorname{ran} t k t=\varnothing$
\end{tabular}

\footnotetext{
${ }^{7}$ In $\mathrm{Z}$ when operations occur outside their preconditions, the post-state is undefined.
} 
Kurbel.STATE denotes the state schema in the class Kurbel, etc.

Firstly, to prove the initialisation correct (US.3) we must prove the following:

$\forall$ Marlowe.STATE; Kurbel.INIT •Ret $\Longrightarrow$ Marlowe.INIT

To do so we must show the following holds (which it clearly does).

$\forall$ mpool $: \mathbb{P}$ Ticket $;$ tkt $:$ Name $\rightarrow$ Ticket $;$ kpool $: \mathbb{P}$ Ticket $;$ bkd $: \mathbb{P}$ Name $\mid b k d=\varnothing \bullet$
$\quad b k d=\operatorname{dom} t k t \wedge k p o o l=$ mpool $\cup$ ran $t k t \wedge$ mpool $\cap \operatorname{ran} t k t=\varnothing \Longrightarrow t k t=\varnothing$

Next, we must show that US.1 holds for the operations Book and Arrive. For the Book operation, this requires us to show that

$\forall$ Kurbel.STATE $\bullet \exists$ Marlowe.STATE $\bullet$ Ret $\wedge$ Pre Marlowe.Book $\Longrightarrow$ Pre Kurbel.Book

This amounts to showing that

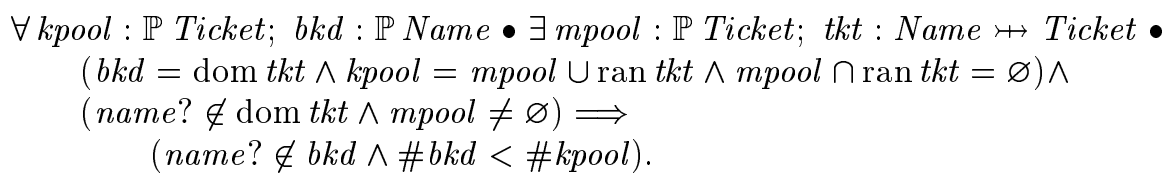

Given the declarations and the constraints in Ret, we proceed as follows.

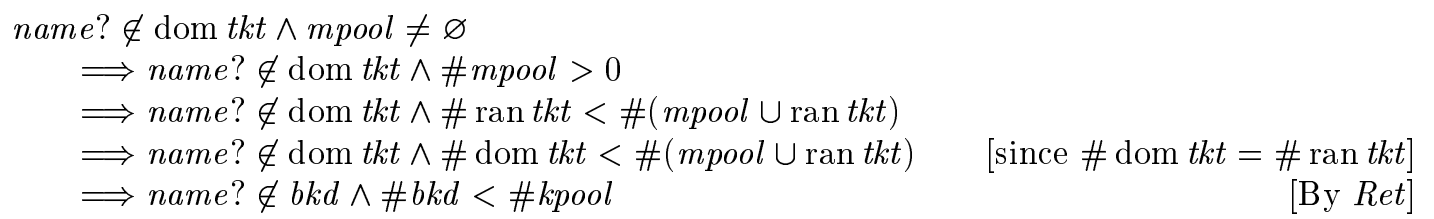

A similar proof can be given for the operation Arrive.

Finally, we must show that US.2 holds for the operations Book and Arrive. For the Arrive operation, this requires us to show that

$$
\begin{aligned}
& \forall \text { Marlowe.STATE }{ }^{\prime}, \text { Kurbel.STATE, Kurbel.STATE' } \bullet \\
& \text { Kurbel.Arrive } \wedge \text { Ret }^{\prime} \Longrightarrow \exists \text { Marlowe.STATE } \bullet \text { Ret } \wedge \text { Marlowe.Arrive } .
\end{aligned}
$$

That is, given the declarations we need to show that

$$
\begin{aligned}
& \left(\text { name } ? \in b k d \wedge b k d^{\prime}=b k d \backslash\{\text { name } ?\} \wedge t ! \in k p o o l \wedge k p o o l^{\prime}=k p o o l \backslash\{t !\} \wedge\right. \\
& \left.b k d^{\prime}=\operatorname{dom} t k t^{\prime} \wedge k p o o l^{\prime}=\text { mpool }^{\prime} \cup \operatorname{ran} t k t^{\prime} \wedge \varnothing=\text { mpool }^{\prime} \cap \operatorname{ran} t k t^{\prime}\right) \Longrightarrow \\
& \exists \text { mpool : } \mathbb{P} \text { Ticket; tkt : Name } \mapsto \text { Ticket } \bullet \\
& (b k d=\operatorname{dom} t k t \wedge k p o o l=m p o o l \cup \operatorname{ran} t k t \wedge m p o o l \cap \operatorname{ran} t k t=\varnothing \wedge \\
& \text { name } \left.? \in \operatorname{dom} t k t \wedge \text { mpool }=\text { mpool }^{\prime} \wedge t k t^{\prime}=\{\text { name } ?\} \quad \forall k t \wedge t !=t k t(\text { name } ?)\right) \text {. }
\end{aligned}
$$

This can be seen to be true if we take mpool $=m p o o l^{\prime}$ and $t k t=t k t^{\prime} \cup\{n a m e ? \mapsto t$ ! $\}$. We only need to prove the first three conjuncts of the consequent, the rest follow trivially from our choice of mpool, etc. For example, with these choices we can then make the following deductions.

$$
\begin{aligned}
\operatorname{dom} t k t & =\operatorname{dom}\left(t k t^{\prime} \cup\{\text { name } ? \mapsto t !\}\right)=\operatorname{dom} t k t^{\prime} \cup\{\text { name } ?\} \\
& =b k d^{\prime} \cup\{\text { name } ?\}=(b k d \backslash\{\text { name? }\}) \cup\{\text { name? }\} \\
& =b k d
\end{aligned}
$$


mpool $\cup \operatorname{ran} t k t=$ mpool $^{\prime} \cup \operatorname{ran}_{t k t} t^{\prime} \cup\{t !\}=k p o o l^{\prime} \cup\{t !\}=k p o o l$

Finally, to show that $m p o o l \cap \operatorname{ran} t k t=\varnothing$ we note that (since $\operatorname{ran} t k t=\operatorname{ran} t k t^{\prime} \cup\{t !\}$ )

$$
\text { mpool } \cap \operatorname{ran} t k t=\left(\text { mpool } \cap \operatorname{ran} t k t^{\prime}\right) \cup \operatorname{mpool} \cap\{t !\}=\varnothing \cup(\text { mpool } \cap\{t !\})
$$

Now from $t ! \in k p o o l \wedge t ! \notin$ kpool $^{\prime}$ we deduce that $t ! \notin m p o o l l^{\prime}=$ mpool. Therefore mpool $\cap$ ran $t k t=$ $\varnothing$.

This concludes the proof that Kurbel is an upward simulation of Marlowe, and therefore a CSP refinement. As with the failures approach, from this we can conclude that BookingSystem ${ }_{K}$ is indeed a refinement of BookingSystem.

\section{Verifying Object-Z and CSP specifications}

This section presents a method of verification for the integrated notation. The method allows us to verify properties of the CSP system specification in terms of its component Object-Z classes. It comprises three phases.

- The first phase involves reasoning about the CSP part of the specification. System properties are stated and transformed to properties of the component Object- $Z$ classes using the notation and laws for CSP operators of [12].

- The properties of the Object-Z classes derived in the first phase will often include terms not readily reasoned about in Object- $Z$. The second phase involves extending the Object$\mathrm{Z}$ classes with auxiliary variables to model these terms. This is achieved using Object-Z inheritance which allows the addition of variables and predicates to the state schema, initial state schema and operations of a class. Reasoning can then be carried out using the logic for Object-Z presented in [19].

- The final phase involves showing that the classes extended with the auxiliary variables are refined by the original Object- $Z$ classes and hence the original classes also satisfy the desired properties.

To illustrate the approach, we will verify the property of BookingSystem stated at the end of Section 2.

\subsection{Reasoning about the CSP processes}

Properties about CSP processes can be stated in term of their failures. Given a process $P$ with failures $F$, the property $\forall(t r, r e f) \in F \bullet S(t r, r e f)$ can be expressed using the notation of [12] as $P$ sat $S(t r, r e f)$. For example, the following property of the process BookingSystem states that the number of bookings made is greater than or equal to the number of tickets allocated to arriving customers.

BookingSystem sat \#tr $\downarrow$ Book $\geqslant \# t r \downarrow$ Arrive

To prove such a property in CSP, we would use the laws for the various CSP operators given in [12]. Therefore, we re-express the property in terms of CSP operators by replacing BookingSystem with its definition in terms of component processes. 


$$
\left(\mid \|_{n: \text { Name }} \text { Customer }_{n}\right) \| \text { Marlowe sat \#tr } \downarrow \text { Book } \geqslant \# t r \downarrow \text { Arrive }
$$

In this form, we can apply the following law for the parallel composition operator ${ }^{8}$.

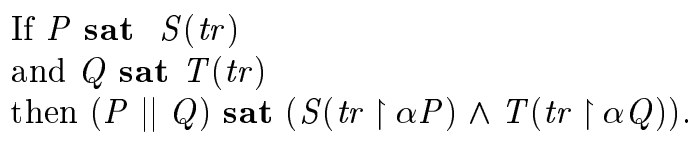

Let $S\left(\operatorname{tr}\left\lceil\alpha\left(\|\|_{n: \text { Name }}\right.\right.\right.$ Customer $\left.\left.{ }_{n}\right)\right)=$ true and, since the alphabet of Marlowe is identical to that of BookingSystem, let $T(\operatorname{tr}\lceil\alpha$ Marlowe $)=\# t r \downarrow$ Book $\geqslant \# t r \downarrow$ Arrive. Using the law for the parallel composition operator, the above property is true whenever the following is true.

Marlowe sat \#tr $\downarrow$ Book $\geqslant \#$ tr $\downarrow$ Arrive

This property is now in terms of a process corresponding to an Object-Z class and we can no longer use the laws for CSP operators. To complete the proof, we require a method for showing the above property is true for the Object-Z class Marlowe.

\subsection{Reasoning about the Object-Z classes}

Building on the work in [26], a logic for reasoning about Object-Z classes is presented in [19]. Properties of classes are expressed as sequents of the form

$$
A:: \quad d \mid \Psi \vdash \Phi
$$

where $A$ is a class name, $d$ is a list of declarations and $\Psi$ and $\Phi$ are lists of predicates. The sequent is valid, i.e. the stated property is true, whenever given the declarations $d$ and predicates $\Psi$ at least one of the predicates in $\Phi$ is true in class $A$. For example, the following is a valid sequent (INIT denotes the declarations and predicates of the INIT schema of Marlowe).

$$
\text { Marlowe :: } \quad \text { INIT } \vdash t k t=\varnothing
$$

The predicates in $\Psi$ and $\Phi$ are only defined in terms of variables and constants which are accessible in the class or declared in $d$. Hence, it is not possible to state properties about sequences of events such as those we would like to prove about the CSP process corresponding to a class. Therefore, we need to introduce auxiliary variables to capture such properties. For example, an auxiliary variable $b k s: \mathbb{N}$ could be added to the class Marlowe to model the CSP term \#tr $\downarrow$ Book. Initially $b k s$ would be zero, it would be incremented each time Book occurs and remain unchanged each time Arrive occurs. Similarly, an auxiliary variable arrs : $\mathbb{N}$ could be added to model the CSP term \#tr $\downarrow$ Arrive.

The addition of such variables to a class is possible using Object-Z inheritance (see [7]). When a class inherits another, schemas from the inherited class are implicitly conjoined with commonnamed schemas in the inheriting class. For example, consider the following class auxMarlowe which inherits Marlowe.

\footnotetext{
${ }^{8}$ As mentioned in [12], this law is valid provided $S$ and $T$ do not mention refusal sets.
} 


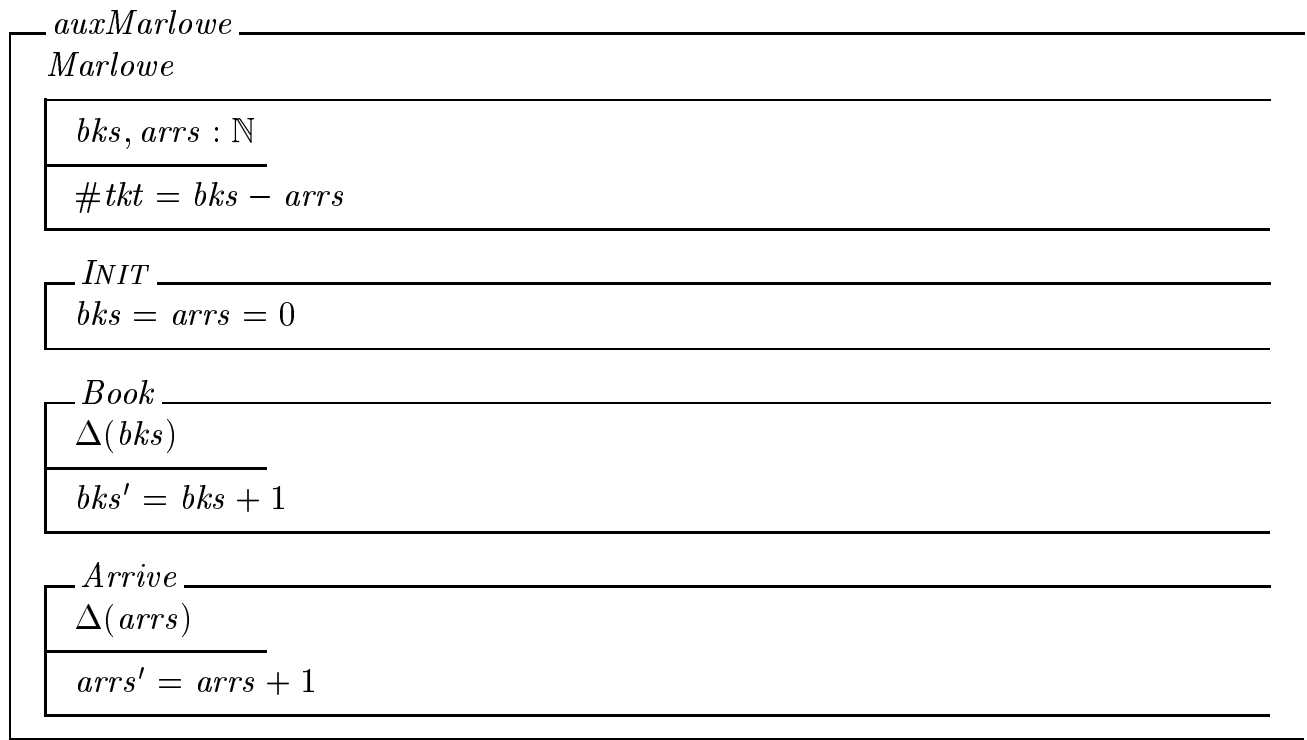

The class auxMarlowe includes all the definitions of class Marlowe and extends them as follows. The state schema has the additional state variables $b k s$ and arrs and the additional predicate $\# t k t=b k s-a r r s$. This predicate isn't strictly necessary but aids the proof of the refinement relation between Marlowe and auxMarlowe as shown in Section 4.3. The initial state schema includes the additional constraint that $b k s$ and arrs are equal to zero and the operations Book and Arrive increment the variables bks and arrs respectively.

To prove the property that the number of bookings is greater than or equal to the number of tickets allocated to arriving customers for the class auxMarlowe, i.e. auxMarlowe sat \#tr $\downarrow$ Book $\geqslant \# t r \downarrow$ Arrive, we need to show that the following sequents are valid.

$$
\begin{aligned}
& \text { auxMarlowe }:: \quad \text { INIT } \vdash \text { bks }=0 \wedge \text { arrs }=0 \\
& \text { auxMarlowe }:: \quad \text { Book } \vdash b k s^{\prime}=b k s+1 \wedge \text { arrs }{ }^{\prime}=\text { arrs } \\
& \text { auxMarlowe }:: \quad \text { Arrive } \vdash b k s^{\prime}=b k s \wedge \text { arrs }{ }^{\prime}=\text { arr }+1 \\
& \text { auxMarlowe }:: \quad \vdash b k s \geqslant \text { arrs }
\end{aligned}
$$

The first three sequents ensure that $b k s$ and arrs model the number of occurrences of the operations Book and Arrive respectively. They can easily be proved using the logic for Object-Z (see [20] for examples of proofs in the logic). The final sequent states the desired property. It can be proved by structural induction, i.e. by proving the following sequents.

$$
\begin{array}{ll}
\text { auxMarlowe }:: & \text { INIT } \vdash \text { bks } \geqslant \text { arrs } \\
\text { auxMarlowe }:: & \text { Book } \vdash \text { bks } \geqslant \text { arrs } \Rightarrow b k s^{\prime} \geqslant \text { arrs }^{\prime} \\
\text { auxMarlowe }:: & \text { Arrive } \vdash b k s \geqslant \text { arr } \Rightarrow b k s^{\prime} \geqslant a r r s^{\prime}
\end{array}
$$

These sequents can also be easily proved using the logic for Object-Z.

The above can be generalised as follows. A property $P$ of a process corresponding to a class $C$ in terms of the number of occurrences of particular events $O p_{1}, \ldots, O p_{n}$,

$$
C \text { sat } P\left(\# t r \downarrow O p_{1}, \ldots, \# t r \downarrow O p_{n}\right)
$$

is true when the following sequents are valid. (The set of operations of the class are $O p_{1}, \ldots, O p_{m}$ where $m \geqslant n$.) 


$$
\begin{aligned}
& C:: \quad I N I T \vdash a_{1}=0 \wedge \ldots \wedge a_{n}=0 \\
& C:: \quad O p_{1} \vdash a_{1}^{\prime}=a_{1}+1 \wedge a_{2}^{\prime}=a_{2} \wedge \ldots \wedge a_{n}^{\prime}=a_{n} \\
& \vdots \\
& C:: \quad O p_{n} \vdash a_{1}^{\prime}=a_{1} \wedge \ldots a_{n-1}^{\prime}=a_{n-1} \wedge a_{n}^{\prime}=a_{n}+1 \\
& C:: \quad O p_{n+1} \vdash a_{1}^{\prime}=a_{1} \wedge \ldots \wedge a_{n}^{\prime}=a_{n} \\
& \vdots \\
& C:: \quad O p_{m} \vdash a_{1}^{\prime}=a_{1} \wedge \ldots \wedge a_{n}^{\prime}=a_{n} \\
& C:: \quad \vdash P\left(a_{1}, \ldots, a_{n}\right)
\end{aligned}
$$

Similarly, we can develop rules for proving other types of properties. For example, a CSP predicate in terms of $O p \in$ ref can be replaced by an Object-Z predicate in terms of $\neg$ pre $O p$ where pre $O p$ denotes the precondition of $O p$. Such rules need to be proved sound. This can be done with respect to the failures semantics of classes presented in Section 2.

\subsection{Proving the refinement relations}

To show that the property proved for auxMarlowe also holds for Marlowe, we need to prove the refinement relation auxMarlowe $\sqsubseteq$ Marlowe. This can be done using the notion of downward simulation defined in Section 3. To do so we first note that the retrieve relation between auxMarlowe and Marlowe is simply the identity (which we denote $I d$ ). Therefore to prove the refinement we have to show that

DS.1 $\forall$ auxMarlowe.STAte; Marlowe.STAte $\bullet$ (Pre auxMarlowe.Book $\Longleftrightarrow$ Pre Marlowe.Book)

DS.2 $\forall$ auxMarlowe.STATE; Marlowe.STATE; Marlowe.STATE' •

Marlowe.Book $\Longrightarrow \exists$ auxMarlowe.STATE' $\bullet$ auxMarlowe.Book

DS.3 $\forall$ Marlowe.INIT $\bullet \exists$ auxMarlowe.INIT $\bullet I d$

together with similar conditions for the operation Arrive. Because we have simply added new state variables under the refinement, these conditions are easily discharged.

DS.1: This amounts to showing that

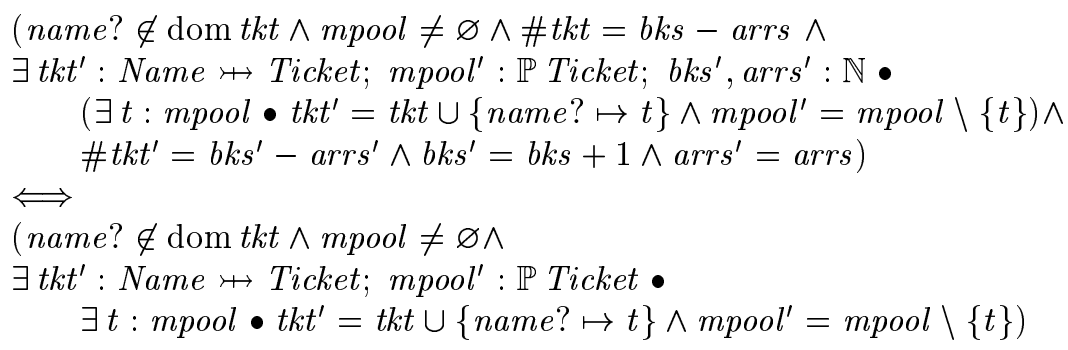

which is easily shown to be true (for example, \#tkt' $=\# t k t+1=b k s-$ arrs $+1=b k s^{\prime}-a^{\prime} r s=$ $\left.b k s^{\prime}-\operatorname{arrs}^{\prime}\right)$.

DS.2: This amounts to showing the following, which again can easily shown to be true.

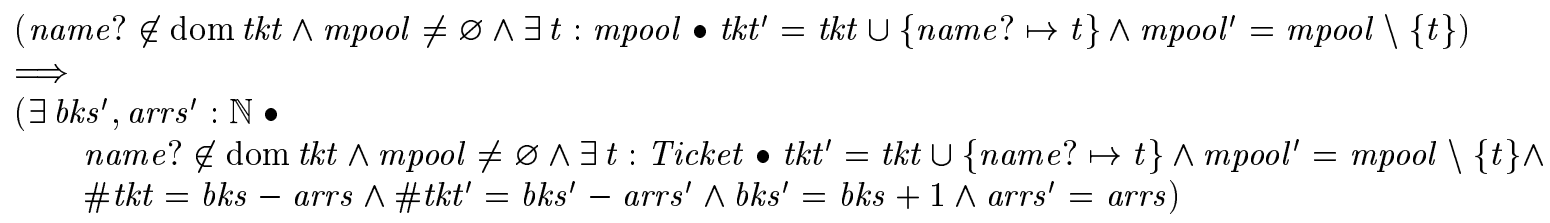


DS.3: To prove this, it is sufficient to show the following, which is easily done.

$$
\forall t k t: \text { Name } \leftrightarrow \text { Ticket } \mid t k t=\varnothing \bullet \exists b k s, \text { arrs }: \mathbb{N} \mid \# t k t=b k s-\text { arrs } \wedge b k s=\text { arrs }=0
$$

The conditions for Arrive can be proved in a similar fashion. Hence, auxMarlowe $\sqsubseteq$ Marlowe. Since we have shown that auxMarlowe sat \#tr $\downarrow$ Book $\geqslant \# t r \downarrow$ Arrive we can deduce that Marlowe sat \#tr $\downarrow$ Book $\geqslant \# t r \downarrow$ Arrive, and hence conclude the proof that the booking system satisfies the desired property. Furthermore, since Marlowe $\sqsubseteq$ Kurbel, we can also conclude that the Kurbel booking system satisfies the property.

\section{Conclusion}

In this paper we have presented methods for refining and verifying specifications written using a combination of Object-Z and CSP. Because we have not modified either of the languages used in the combined notation, we have been able to use existing methods in our approach to refinement and verification in the combined notation. For example, by giving Object-Z classes a CSP semantics, we can use CSP refinement as the refinement relation for the integrated notation. A refinement can be verified by either calculating the failures semantics directly, or by applying standard state-based refinement relations to the Object- $Z$ components.

To verify behavioural properties of the CSP system specification we use the Object-Z logic to prove subsidiary properties of the Object- $Z$ component classes, these properties are then combined by application of CSP laws to deduce the desired behavioural properties of the overall system.

Some further areas of work remain. In particular, in addition to the state-based methods of refinement presented above, further methods of refinement need to be developed for specifications whose system structure changes under the refinement. For example, how can one verify the refinement of the Object-Z Kurbel class in the example presented above into two or more communicating Object-Z classes without having to resort to calculation of their semantics? Section 4.2 developed an approach for reasoning about the Object-Z classes in a combined specification, and presented rules for verifying certain properties. Further verification rules for a range of other types of properties need to be developed, and these need to be proved sound with respect to the Object-Z logic and the failures semantics developed in this paper.

\section{References}

[1] T. Bolognesi and E. Brinksma. Introduction to the ISO specification language LOTOS. Computer Networks and ISDN Systems, 14(1):25-59, 1988.

[2] S.D. Brookes, C.A.R. Hoare, and A.W. Roscoe. A theory of communicating sequential processes. Journal of the ACM, 31(3):560-599, 1984.

[3] S.D. Brookes and A.W. Roscoe. An improved failures model for communicating processes. In Pittsburgh Symposium on Concurrency, volume 197 of Lecture Notes in Computer Science, pages 281-305. Springer-Verlag, 1985.

[4] J. Derrick, E.A.Boiten, H. Bowman, and M. Steen. Supporting ODP - translating LOTOS to Z. In First IFIP International workshop on Formal Methods for Open Object-based Distributed Systems. Chapman \& Hall, 1996.

[5] J. Dong, R. Duke, and G. Rose. An object-oriented approach to the semantics of programming languages. In G. Gupta, editor, 17th Annual Computer Science Conference (ACSC'17), pages $767-775,1994$. 
[6] D. Duke and R. Duke. Towards a semantics for Object-Z. In D. Bjorner, C.A.R. Hoare, and H. Langmaack, editors, VDM'90:VDM and Z!, volume 428 of Lecture Notes in Computer Science, pages 242-262. Springer-Verlag, 1990.

[7] R. Duke, G. Rose, and G. Smith. Object-Z: A specification language advocated for the description of standards. Computer Standards and Interfaces, 17:511-533, 1995.

[8] M. Nielsen et al. The RAISE language, methods and tools. Formal Aspects of Computing, $1: 85-114,1989$.

[9] C. Fischer. Combining CSP and Z. Submitted to Formal Methods Europe (FME '97), 1997.

[10] J. He. Process refinement. In J. McDermid, editor, The Theory and Practice of Refinement. Butterworths, 1989.

[11] M. Heisel and C. Sühl. Formal specification of safety-critical software with Z and real-time CSP. In E. Schoitsch, editor, Proceedings 15th International Conference on Computer Safety, Reliability and Security, pages 31-45. Springer, 1996.

[12] C.A.R. Hoare. Communicating Sequential Processes. International Series in Computer Science. Prentice-Hall, 1985.

[13] ITU Recommendation X.901-904. Open Distributed Processing - Reference Model - Parts 1-4, July 1995.

[14] C.B. Jones. Systematic Software Development using VDM. International Series in Computer Science. Prentice-Hall, 1986.

[15] M.B. Josephs. A state-based approach to communicating processes. Distributed Computing, 3:9-18, 1988.

[16] R. Milner. Communication and Concurrency. International Series in Computer Science. Prentice-Hall, 1989.

[17] A.W. Roscoe. An alternative order for the failures model. Journal of Logic and Computation, 3(2), 1993.

[18] A.W. Roscoe. Unbounded nondeterminism in CSP. Journal of Logic and Computation, 3(2), 1993.

[19] G. Smith. Extending $\mathcal{W}$ for Object-Z. In J. Bowen and M. Hinchey, editors, 9th International Conference of $Z$ Users, volume 967 of Lecture Notes in Computer Science, pages 276-295. Springer-Verlag, 1995.

[20] G. Smith. Formal verification of Object-Z specifications. Technical Report 95-55, Software Verification Research Centre, Department of Computer Science, University of Queensland, Australia, 1995.

[21] G. Smith. A fully abstract semantics of classes for Object-Z. Formal Aspects of Computing, $7(3): 289-313,1995$.

[22] G. Smith. A semantic integration of Object-Z and CSP for the specification of concurrent systems. To appear in Formal Methods Europe (FME '97), 1997.

[23] J.M. Spivey. The Z Notation: A Reference Manual (2nd Ed.). International Series in Computer Science. Prentice-Hall, 1992.

[24] M. Weber. Combining Statecharts and Z for the design of safety-critical systems. In M.-C. Gaudel and J.C.P. Woodcock, editors, FME '96 - Industrial Benefits and Advances in Formal Methods, volume 1051 of Lecture Notes in Computer Science, pages 307-326. Springer-Verlag, 1996. 
[25] J. Woodcock and J. Davies. Using Z: Specification, Refinement, and Proof. International Series in Computer Science. Prentice-Hall, 1996.

[26] J.C.P. Woodcock and S.M. Brien. $\mathcal{W}$ : A logic for Z. In J.E. Nicholls, editor, Z User Workshop, Workshops in Computing, pages 77-98. Springer-Verlag, 1992.

[27] J.C.P. Woodcock and C.C. Morgan. Refinement of state-based concurrent systems. In D. Bjorner, C.A.R. Hoare, and H. Langmaack, editors, VDM'90:VDM and Z!', volume 428 of Lecture Notes in Computer Science. Springer-Verlag, 1990. 